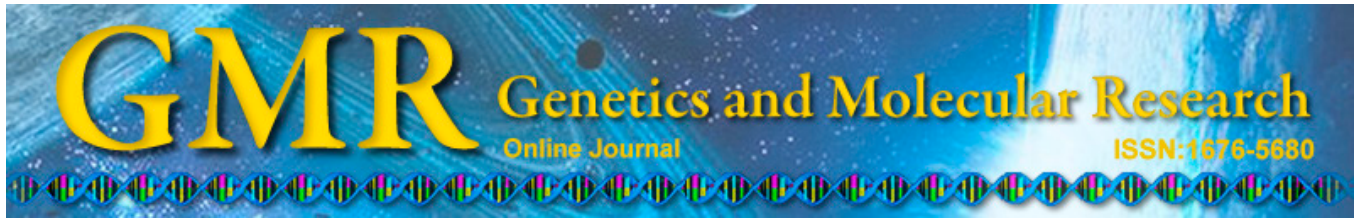

\title{
Myocardial protective effects of Munziq in myocardial ischemia-reperfusion injury rats with abnormal savda syndrome
}

\author{
M. Abudunaibi ${ }^{1}$, A. Mulati ${ }^{1}$, S. Aisikaer ${ }^{1}$, M. Zulifeiya ${ }^{1}$, J. Qiao ${ }^{1}$, \\ M. Gulibositan ${ }^{2}$, A. Aili ${ }^{1}$ and U. Halmurat ${ }^{3}$ \\ ${ }^{1}$ Department of Cardiac Surgery, \\ First Teaching Hospital of Xinjiang Medical University, Urumqi, \\ Xinjiang, China \\ ${ }^{2}$ Otolaryngology Department of Xinjiang Autonomous Region People's Hospital, \\ Xinjiang, China \\ ${ }^{3}$ Uyghur Medicine Institute of Xinjiang Medical University, Xingiang, China \\ Corresponding author: A. Mulati \\ E-mail: nebi-boss@hotmail.com/nebi_boss@126.com
}

Genet. Mol. Res. 14 (2): 3426-3435 (2015)

Received June 3, 2014

Accepted October 22, 2014

Published April 15, 2015

DOI http://dx.doi.org/10.4238/2015.April.15.6

\begin{abstract}
This study evaluated the influence and mechanism of different Munziq doses on myocardial ischemia reperfusion injury (MIRI) rats with abnormal savda. Wistar rats $(\mathrm{N}=96)$ were divided into the following 8 groups (12 rats each): ischemia-reperfusion (I/R) model group, high-dose, medium-dose, and low-dose Munziq groups, normal I/R group, sham model group, normal sham group, and Atorvastatin group. Changes in heart physiology and myocardial morphology after injury with MIRI were monitored in each group. Heat shock protein 70 (HSP70) and calcitonin gene-related peptide (CGRP) protein expression and serum concentrations of superoxide dismutase (SOD), malondialdehyde (MDA), interleukin (IL)-6, and IL-8 were detected by using western blot and ELISA methods, respectively. The large-dose Munziq group showed the most significant changes. The VPC incurring
\end{abstract}


time was not delayed in the small-dose group, but the accumulative time was significantly reduced $(\mathrm{P}<0.01)$. The ventricular tachycardia incurring time did not differ significantly between groups. Compared with the normal sham surgical group, the I/R groups showed significant increases in HSP70 and CGRP expression $(\mathrm{P}<0.01)$ and MDA, IL-6, and IL-8 concentrations $(\mathrm{P}<0.05)$ and a significant decrease in the SOD concentration $(\mathrm{P}<0.05)$. Compared with the $\mathrm{I} / \mathrm{R}$ groups, Munziq intervention significantly enhanced HSP70 and CGRP expression $(\mathrm{P}<$ 0.01 ), significantly decreased MDA, IL- 6 , and IL-8 concentrations ( $\mathrm{P}$ $<0.05)$, and significantly increased the SOD concentration $(\mathrm{P}<0.05)$. In conclusion, Munziq intervention improves cardiac physiological function, increases the expression of HSP70 and CGRP, and decreases the inflammatory reaction in MIRI model rats.

Key words: Munziq; Abnormal savda; MIRI; Heat shock proteins; Inflammatory reaction

\section{INTRODUCTION}

Myocardial ischemia/reperfusion injury (MIRI) occurs when the myocardial blood supply is briefly suspended and then recovered, resulting in damage to the ischemic myocardium (Schulze et al., 2007). The mechanism of MIRI is not completely understood, and many factors that are affected by this process may contribute to increasing the severity of the damage after reperfusion. Presently, as the physiology of heart diseases is gradually being investigated in depth, studies on myocardial protection methods have become more detailed and complex. With the promotion and application of technologies like heart surgical extracorporeal circulation, coronary artery bypass surgery, complicated congenital heart disease cardiac surgery, cardiac valve replacement, and major vascular surgery, MIRI has become the main factor that interferes with the effect of cardiac vascular surgery therapy (Lum and Roebuck, 2005). The mechanism of MIRI may involve many factors, such as oxygen free radicals (OFR), calcium overload, myocardial fiber energy metabolism disorders, vascular endothelial cells, nitric oxide, heat shock proteins (HSPs), neutrophilic granulocytes, and apoptosis, and all of these factors may contribute to the pathology of MIRI (Hoffman et al., 2007; Ruiz-Gines et al., 2006). Ischemia, hypoxia, reperfusion, and mechanical damage may occur during open-heart surgery, resulting in a series of pathological changes to the myocardium. Therefore, the issue of myocardium protection is especially important.

The humoral theory of Uygur medicine states that, when various adverse factors (i.e., adverse environment, diet, life style, and mental factors) influence the human body over a long period, 1 or more of the 4 normal body fluids (savda, phlegm, blood, and sapra) that maintain the normal physiological functions of the human body "burn" to generate pathological products with different characteristics (abnormal savda, abnormal phlegm, abnormal blood, and abnormal sapra). The generation of these pathological products results in an imbalance of the 4 body fluids and leads to abnormalities in nutrient function, transformation, and metabolism due to changes in nutrients and their characteristics in body fluids. Accordingly, cells, tissues, and organs absorb fewer nutrients because the absorption process becomes difficult or they absorb nutrients with abnormal characteristics and qualities; this causes changes in the charac- 
teristics of the cells, tissues, including vessel wall tissue, and organs, resulting in disturbances in normal physiological functions. Meanwhile, owing to the decrease of various forces (absorption force, gripping force, driving force, and excretion force) and changes in physical characteristics (increased mass, decreased temperature, and enhanced stickiness) of abnormal body fluids, the body fluids move at a slower speed. This is likely to produce deposits on the vessel wells, which thickens the vessel walls, decreases their elasticity, and makes the lumen narrower. Further, the deposits generated on the vessel wall act as a source of simulation, aggravating the disturbance of the vessel wall tissue and cell function, and this results in cardiovascular diseases like atherosclerosis, hypertension, and coronary heart disease (Hamulati, 2008).

Previous studies have shown that the introduction of abnormal savda Munziq can effectively improve the general status of animal models induced with abnormal savda vector and has some protective effects on myocardial damage. Generally, most diseases that occur following cardiac surgery are associated with abnormal savda symptoms. Thus, it is necessary to establish a MIRI model based on abnormal savda in order to perform myocardium protection studies under abnormal savda conditions, investigate abnormal savda characteristics and disease mechanisms, and determine the mechanism of the effect of Munziq. Accordingly, this enables the exploration of methods for preventing, curing, and intervening in cardiac diseases with abnormal savda characteristics.

\section{MATERIAL AND METHODS}

\section{Model establishment}

The MIRI animal model in animals with abnormal savda was established according to the method of Hamulati et al. (2006).

\section{Experimental animals and grouping}

Experimental animals (96 rats without gender limitation) were provided by the Animal Research Center of the First Affiliated Hospital of Xinjiang Medical University. These 96 rats with abnormal savda were divided into the following 8 groups (12 rats per group): ischemia-reperfusion (I/R) model group, high-dose Munziq group, medium-dose Munziq group, low-dose Munziq group, normal I/R group, sham model group, normal sham group, and Atorvastatin group.

\section{Methods}

\section{Munziq dosing method}

One hour before the experiment on each day, abnormal savda Munziq was intragastrically administered to the rats in the Munziq groups. The low-dose group received $2.53 \mathrm{~g} / \mathrm{kg}$ ( $2.5 \mathrm{~g}$ of Munziq dissolved in $1 \mathrm{~mL}$ distilled water, at an aquatic dose of $8.58 \mathrm{~g} / \mathrm{kg}$ ), which is equivalent to the effective clinical dose. The medium-dose group received $5.069 \mathrm{~g} / \mathrm{kg}(5.0$ $\mathrm{g}$ of Munziq dissolved in $1 \mathrm{~mL}$ distilled water at a raw dose of $17.16 \mathrm{~g} / \mathrm{kg}$ ), which is double the effective clinical dose. The high-dose group received $10.12 \mathrm{~g} / \mathrm{kg}(10 \mathrm{~g}$ of abnormal savda Munziq dissolved in $1 \mathrm{~mL}$ distilled water at a raw dose of $34.32 \mathrm{~g} / \mathrm{kg}$ ), which is equivalent to 5 times the effective clinical dose. For every $100 \mathrm{~g}$, rats were given $1.5 \mathrm{~mL}$ Munziq solution. 
After 21 days of administration, the general biological characteristics of the experimental animals were observed, such as body temperature, body weight, food intake, water intake, tongue appearance, and urine and stool characteristics.

\section{I/R method}

To establish the I/R model, the rats were intraperitoneally anesthetized with $30 \mathrm{~g} / \mathrm{L} 30$ $\mathrm{mg} / \mathrm{kg}$ sodium pentobarbital. The animals were placed on a small animal operating table in the supine position, and their limbs were subcutaneously punctured and connected to an electrocardiogram (ECG) machine (Carewell compamy, Shenzhen, China). After 5 min, a normal ECG was recorded (paper speed, $100 \mathrm{~mm} / \mathrm{s}$; voltage, $20 \mathrm{~mm} / \mathrm{mV}$ ) as the control. A tracheostomy was performed for ventilator-assisted breathing. The ventilator parameters were as follows: respiratory rate, 50 beats $/ \mathrm{min}$; tidal volume, $20 \mathrm{~mL} / \mathrm{kg}$, and breathing ratio, 1:1 (Krenz and Robbins, 2004). The 3rd and 4th ribs were cut, the chest wall was opened with a wire retractor, a pair of lines was inserted through both sides of the chest wall muscle, and a small circle was left inside the muscle. The pericardium was carefully cut, the heart was exposed, and a cotton swab was lightly placed on the heart. The left coronary vein was selected as the marker, and a 520 noninvasive suture needle was inserted in 3 to $4 \mathrm{~mm}$ of the lower edge of the left atrial appendage at an approximate depth of $115 \mathrm{~mm}$. The needle was pulled out obliquely in the top right of the pulmonary cone direction, with a stitch length of 3 to $4 \mathrm{~mm}$. A fluted latex tube with a $115-\mathrm{mm}$ inner diameter and a $0.15-\mathrm{cm}$ length was set for the standby, and both ends of the line were pulled through the small circle. The line was tightened and was ligated with a latex tube 10 minutes after initiation of steady breathing. The latex tube was compressed with the left anterior descending coronary artery (LAD). The multi-lead ECG was used for recording throughout the experiment. After $30 \mathrm{~min}$ of coronary artery ligation, the ligature was released to restore blood perfusion.

\section{Detection methods}

The mean arterial pressure (MAP) and heart rate (HR) were recorded after 15 min of cardiac stabling (baseline), immediately before ischemia, after $30 \mathrm{~min}$ of ischemia, and after 120 min of reperfusion. Femoral venous blood $(4-5 \mathrm{~mL})$ was sampled at reperfusion for 120 min to detect blood indicators. After reperfusion for $120 \mathrm{~min}$, the rats were killed and the myocardial tissue was sampled for protein indicator detection.

\section{Influence of different Munziq intervention doses on rat cardiac physiology}

After the rats were dosed, open chest surgery and the LAD ligation operation were performed as described for the I/R group. The MAP, HR, Real Portolio Project (MAP x HR) and ECG at the I/R stage were observed by using the BL-4205 biological experimental system.

\section{Statistical methods}

All measurement data are reported as means \pm standard deviation. One-way ANOVA was used to compare the means of the groups. If homogeneity of variance was observed, the comparison between each group was conducted by using the least significant difference method. If heterogeneity of variance was observed, the comparison between each 
group was conducted by using the rank test. The rates of each group were compared by using the chi-square test or the Fisher exact test on counting data. P values $<0.05$ were considered to be significantly different. All statistical analyses were conducted by using SPSS17.0.

\section{RESULTS}

\section{Influence of Munziq intervention on rat MAP and HR at the reperfusion stage}

Compared with before ischemia, the MAP of each group decreased at the instant of LAD ligation, with the I/R group exhibiting a faster and larger decrease $(\mathrm{P}<0.05)$. The MAP of each group recovered after $30 \mathrm{~min}$ of ischemia, with the I/R group still showing a faster and larger increase $(\mathrm{P}<0.05)$. In the reperfusion stage, the MAP of each group showed stability with a small increase, except for the I/R group, which showed a continuous decrease; at 120 min of reperfusion, the MAP of each group was stable at the before-ischemia level, except for the I/R group, which showed a continuous decline (Table 1).

Before ischemia, the HRs of the Munziq groups were significantly lower than those of the I/R group $(\mathrm{P}<0.05)$. No significant difference was observed between each group after the ischemia period began (Table 2).

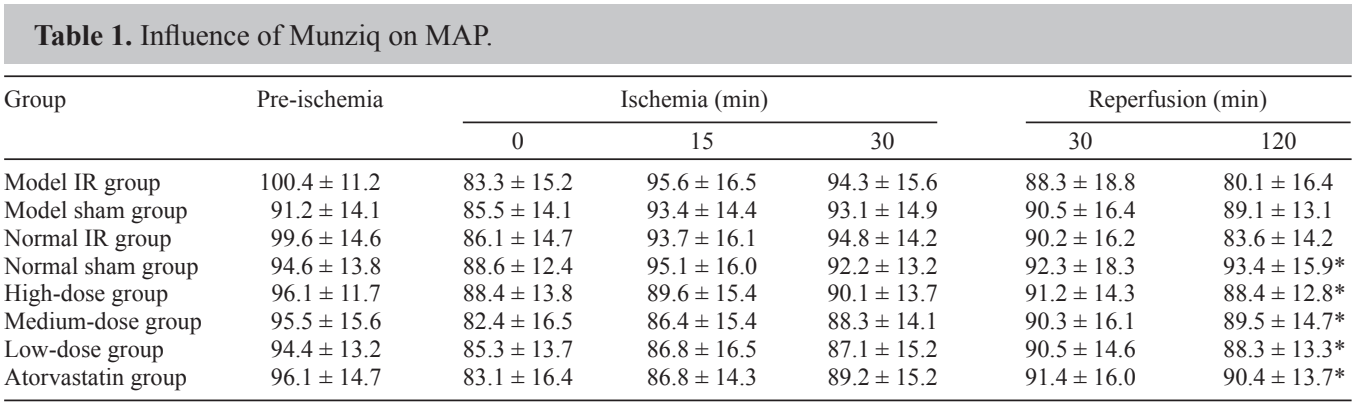

Table 2. Influence of Munziq on HR.

\begin{tabular}{|c|c|c|c|c|c|c|}
\hline \multirow[t]{2}{*}{ Group } & \multirow[t]{2}{*}{ Pre-ischemia } & \multicolumn{3}{|c|}{ Ischemia (min) } & \multicolumn{2}{|c|}{ Reperfusion (min) } \\
\hline & & 0 & 15 & 30 & 30 & 120 \\
\hline Model IR group & $361 \pm 44$ & $348 \pm 48$ & $345 \pm 39$ & $337 \pm 41$ & $313 \pm 32$ & $297 \pm 47$ \\
\hline Model sham group & $358 \pm 49$ & $355 \pm 42$ & $345 \pm 48$ & $347 \pm 48$ & $333 \pm 39$ & $324 \pm 55$ \\
\hline Normal IR group & $360 \pm 48$ & $344 \pm 41$ & $332 \pm 37$ & $330 \pm 46$ & $302 \pm 38$ & $298 \pm 48$ \\
\hline Normal sham group & $349 \pm 43$ & $347 \pm 41$ & $338 \pm 51$ & $336 \pm 47$ & $331 \pm 32$ & $309 \pm 50$ \\
\hline High-dose group & $301 \pm 37^{*}$ & $313 \pm 41$ & $318 \pm 29$ & $321 \pm 37$ & $319 \pm 34$ & $323 \pm 51 *$ \\
\hline Medium-dose group & $311 \pm 35^{*}$ & $326 \pm 49$ & $312 \pm 38$ & $315 \pm 39$ & $324 \pm 39$ & $319 \pm 48 *$ \\
\hline Low-dose group & $308 \pm 38^{*}$ & $310 \pm 38$ & $316 \pm 30$ & $319 \pm 33$ & $322 \pm 39$ & $318 \pm 38^{*}$ \\
\hline Atorvastatin group & $311 \pm 35^{*}$ & $326 \pm 49$ & $312 \pm 38$ & $315 \pm 39$ & $324 \pm 39$ & $319 \pm 48^{*}$ \\
\hline
\end{tabular}

Compared to pre-ischemia, ${ }^{*} \mathrm{P}<0.05$.

\section{Influence of Munziq pretreatment on the ECG of rats at the I/R stage}

The ST segment of each group began to increase at the instant of ischemia, with the I/R group showing the fastest and largest increase. The ST segment of each group reached a peak at $30 \mathrm{~min}$ of ischemia. Compared with the I/R group, the Munziq groups (high-dose, 
medium-dose, and low-dose) showed a significantly slower and smaller increase $(\mathrm{P}<0.05)$. The ST segment of each group showed a large decline at 30 min of reperfusion, which stabilized to the initial level at $120 \mathrm{~min}$ (Table 3).

Table 3. Influence of Munziq pretreatment on the ECG of rats at the I/R stage.

\begin{tabular}{|c|c|c|c|c|c|c|}
\hline \multirow[t]{2}{*}{ Group } & \multirow[t]{2}{*}{ Pre-ischemia } & \multicolumn{3}{|c|}{ Ischemia (min) } & \multicolumn{2}{|c|}{ Reperfusion (min) } \\
\hline & & 0 & 15 & 30 & 30 & 120 \\
\hline Model IR group & $0.03 \pm 0.02$ & $0.33 \pm 0.05$ & $0.44 \pm 0.16$ & $0.50 \pm 0.11$ & $0.12 \pm 0.07$ & $0.05 \pm 0.06$ \\
\hline Model sham group & $0.03 \pm 0.02$ & $0.05 \pm 0.04$ & $0.08 \pm 0.06$ & $0.07 \pm 0.04$ & $0.06 \pm 0.03$ & $0.04 \pm 0.04$ \\
\hline Normal IR group & $0.04 \pm 0.03$ & $0.24 \pm 0.07$ & $0.33 \pm 0.11$ & $0.40 \pm 0.13$ & $0.10 \pm 0.05$ & $0.07 \pm 0.03$ \\
\hline Normal sham group & $0.04 \pm 0.03$ & $0.06 \pm 0.05$ & $0.07 \pm 0.05$ & $0.08 \pm 0.06$ & $0.06 \pm 0.05$ & $0.05 \pm 0.04$ \\
\hline High-dose group & $0.03 \pm 0.03$ & $0.15 \pm 0.04 *$ & $0.18 \pm 0.10^{*}$ & $0.22 \pm 0.08 *$ & $0.08 \pm 0.05$ & $0.04 \pm 0.02$ \\
\hline Medium-dose group & $0.02 \pm 0.01$ & $0.16 \pm 0.07 *$ & $0.15 \pm 0.08^{*}$ & $0.18 \pm 0.07^{*}$ & $0.05 \pm 0.04$ & $0.04 \pm 0.03$ \\
\hline Low-dose group & $0.03 \pm 0.02$ & $0.23 \pm 0.15^{*}$ & $0.28 \pm 0.13^{*}$ & $0.34 \pm 0.10^{*}$ & $0.09 \pm 0.06$ & $0.04 \pm 0.04$ \\
\hline Atorvastatin group & $0.02 \pm 0.02$ & $0.15 \pm 0.06^{*}$ & $0.16 \pm 0.07^{*}$ & $0.19 \pm 0.08^{*}$ & $0.05 \pm 0.04$ & $0.04 \pm 0.02$ \\
\hline
\end{tabular}

Compared to pre-ischemia, $* \mathrm{P}<0.05$.

\section{Influence of Munziq pretreatment on HSP70 and calcitonin gene-related peptide in rats at the $I / R$ stage}

The expression of $\beta$-actin did not differ significantly among the groups. However, the expression levels of HSP70 and calcitonin gene-related peptide (CGRP) were significantly higher in the $\mathrm{I} / \mathrm{R}$ group than in the normal sham group $(\mathrm{P}<0.01)$. Further, the protein levels of HSP70 and CGRP were significantly higher in the Munziq groups than in the I/R group (P $<0.01)$. The expression of HSP70 increased and the expression of CGRP decreased as the amount of Munziq increased (Figure1).

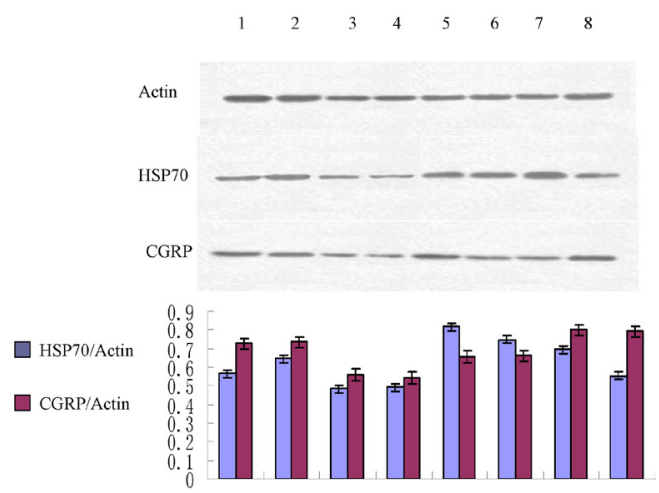

Figure 1. Influence of Munziq pretreatment on HSP70 and CGRP protein expression (western blotting): Representative western blot images are shown in the upper panel. Densiometric analysis of the protein bands are shown in the lower panel. Lane $1=$ model IR group; lane 2 = model sham group; lane 3 = normal IR group; lane 4 $=$ normal sham group; lane $5=$ high-dose Munziq group; lane $6=$ medium-dose Munziq group; lane $7=$ low-dose Munziq group; lane $8=$ atorvastatin group.

\section{The influence of Munziq pretreatment on serum malondialdehyde and superoxide dismutase concentrations}

Compared to the sham group, the malondialdehyde (MDA) concentration was significantly increased and the superoxide dismutase (SOD) concentration was 
significantly decreased in the $\mathrm{I} / \mathrm{R}$ group $(\mathrm{P}<0.01)$. Regardless of dose, the SOD concentrations were significantly increased $(\mathrm{P}<0.05)$ and the MDA concentrations were significantly decreased $(\mathrm{P}<0.05)$ in the Munziq pretreatment groups compared to the $I / R$ group. However, the SOD concentration was higher and the MDA concentration was lower in both the high-dose and medium-dose groups than in the low-dose group. The SOD and MDA concentrations did not differ significantly between the medium-dose group and high-dose group. Compared to the I/R group, the SOD concentration was increased and the MDA concentration was decreased in the Atorvastatin group. The MDA and SOD concentrations were significantly different between the Munziq pretreatment groups and the Atorvastatin group (Table 4).

Table 4. Influence of Munziq on MDA and SOD levels.

\begin{tabular}{lcr}
\hline Groups & MDA $(\mathrm{nmol} / \mathrm{mL})$ & SOD $(\mathrm{nmol} / \mathrm{mL})$ \\
\hline Model IR group & $4.83 \pm 0.08^{*}$ & $99.32 \pm 4.45^{*}$ \\
Model sham group & $2.23 \pm 0.09 \triangle *$ & $110.05 \pm 4.05^{* \Delta}$ \\
Normal IR group & $4.31 \pm 0.04$ & $101.12 \pm 3.35^{* \Delta}$ \\
Normal sham group & $2.31 \pm 0.09 \triangle *$ & $136.22 \pm 3.15^{\triangle}$ \\
High-dose group & $2.53 \pm 0.07 \triangle *$ & $128.15 \pm 4.14^{* \Delta}$ \\
Medium-dose group & $2.66 \pm 0.11^{\triangle *}$ & $125.16 \pm 4.07^{* \Delta}$ \\
Low-dose group & $2.94 \pm 0.12^{\triangle *}$ & $114.23 \pm 3.15^{* \Delta}$ \\
Atorvastatin group & $2.83 \pm 0.11^{\triangle *}$ & $121.14 \pm 4.16^{* \Delta}$ \\
\hline
\end{tabular}

Compared to normal sham group, ${ }^{*} \mathrm{P}<0.05$; Compared to Model IR group, ${ }^{\triangle} \mathrm{P}<0.05$.

\section{Influence of Munziq and Atorvastatin pretreatment on serum IL-6 and IL-8 con- centrations}

The concentrations of IL- 6 and IL- 8 were significantly increased $(\mathrm{P}<0.01)$ in the I/R group compared to the sham group. The IL- 6 and IL- 8 concentrations were significantly lower in the Munziq pretreatment groups, regardless of dose, than in the $\mathrm{I} / \mathrm{R}$ group $(\mathrm{P}<$ $0.05)$. The IL- 8 concentrations were not different between the Munziq dose groups. However, the IL-6 concentration was significantly different between the low-dose group and the high-dose group $(\mathrm{P}<0.01)$. Compared to the I/R group, the IL- 6 and IL-8 concentrations were decreased significantly in the Atorvastatin group $(\mathrm{P}<0.01)$. The IL-6 and IL-8 concentrations were not significantly different between the Munziq pretreatment groups and the Atorvastatin group (Table 5).

\section{Table 5. Influence of Munziq on IL-6 and IL-8 levels.}

\begin{tabular}{lcc}
\hline Groups & $\mathrm{IL}-6(\mathrm{pg} / \mathrm{mL})$ & $\mathrm{IL}-8(\mathrm{pg} / \mathrm{mL})$ \\
\hline Model IR group & $284.83 \pm 5.28^{*}$ & $699.32 \pm 12.45^{*}$ \\
Model sham group & $92.23 \pm 2.09^{\triangle *}$ & $510.05 \pm 12.05^{* \Delta}$ \\
Normal IR group & $244.31 \pm 4.04$ & $601.12 \pm 13.35^{* \Delta}$ \\
Normal sham group & $82.31 \pm 3.09 \triangle *$ & $436.22 \pm 11.15^{\triangle}$ \\
High-dose group & $122.53 \pm 3.07 \Delta *$ & $499.15 \pm 14.14^{* \Delta}$ \\
Medium-dose group & $166 \pm 4.11^{\triangle *}$ & $501.16 \pm 14.07^{* \Delta}$ \\
Low-dose group & $194 \pm 0.12^{\triangle *}$ & $514.23 \pm 13.15^{* \Delta}$ \\
Atorvastatin group & $183 \pm 3.12^{\triangle *}$ & $501.12 \pm 14.15^{* \Delta}$ \\
\hline
\end{tabular}

Compared to normal IR group, ${ }^{*} \mathrm{P}<0.05$; Compared to Model IR group, $\triangle \mathrm{P}<0.05$. 


\section{DISCUSSION}

This study revealed that Munziq has an obvious protective effect on the savda rat MIRI model. The HSP70 expression level tended to rise as the Munziq dosage increased. In addition, the levels of inflammatory factors significantly decreased after Munziq pretreatment, suggesting that the myocardial protective effect of Munziq is due to an anti-inflammatory effect.

Previous studies indicated that abnormal savda Munziq in traditional Uygur medicine has antioxidant effects (Abdiryim et al., 2011) and can protect DNA from oxidative damage (Yusup et al., 2004). Abnormal savda Munziq was shown to have a protective effect against the membrane lipid peroxidation damage caused by free radicals and to enhance the activities of SOD and $\mathrm{Ca}^{2+}-\mathrm{Mg}^{2+}$-ATPase in the mitochondrion, maintaining the integrity of the mitochondrion membrane structure (Abdiryim et al., 2012). These studies show that abnormal savda Munziq decreases free radical damage to the mitochondrion, has a protective effect on mitochondrion structure and function, accordingly maintaining the integrity of mitochondrion structure and function, and plays a role in preventing and curing difficult diseases like tumors, asthma, diabetes, hypertension, and coronary heart disease at the subcellular level (Hamulati et al., 2006). Previous studies showed that the vacuolar degeneration degree of myocardial cells in the abnormal savda Munziq intervention group was improved compared with the model group. Notably, the degree of vacuolar degeneration of myocardial cells was obviously improved in the small-dose and medium-dose Munziq groups.

Hamulati et al. (2006), who considered abnormal savda to be the core cause of complicated diseases like tumors, hypertension, diabetes, and severe asthma, established an abnormal savda vector animal model to replicate immune disease, tumors, and diabetes through multiple factors having complex effects according to Uygur medicine theory. These models imitated the performance of abnormal savda, and made the systemic evaluation of the model possible in a feasible and repeatable manner. By performing multiple systemic studies on the models, the feasibility and repeatability of the model was proven through the obtainment of histological and molecular/genetic data, thereby providing an effective and scientific research platform for respiratory system diseases, immune system diseases, and tumors from the perspective of Uygur medicine. Further detailed studies are required regarding the effect of abnormal savda on the cardiovascular system.

HSPs, also called stress proteins, are endogenous protective proteins produced by an organism or isolated cells in culture under stress. Presently, HSP70 is the most well-studied protein in the HSP family. Recent studies have shown that HSP70 is expressed in the I/R myocardium, which has a protective effect on the myocardium via multiple mechanisms. Myocardial ischemia is a common stress reaction that induces myocardial cells to express HSP70. Several reasons have been proposed for the generation of HSP70 during MIRI: 1) OFR generation causes disordered protein metabolism, and a substrate of this molecular reaction activates heat shock factor protein 1, which induces HSP70 mRNA gene expression; 2) HSP70 induced by heat stress has a protective effect against I/R damage; and 3) under stress, HSP70 is beneficial for eliminating and repairing degenerated proteins by stabilizing these degenerated protein in cells, thereby playing a role in protecting and recovering cell function under stress (Sun et al., 2005; Li and Lv, 2011). CGRP co-reacts with substance P and neurokinin A, which is a neuropeptide widely distributed in the central and peripheral nervous systems, to mediate neurogenic inflammation (Huang et al., 2009). CGRP participates in the pathological process of myocardial ischemia in the myocardium. 
In addition, the effects of OFR in the process of MIRI have been demonstrated. MDA is produced after OFR-induced lipid peroxidation; MDA has strong cross-linking ability, which can lead to the inactivation of several enzymes. Therefore, the MDA level is often used to reflect the degree of cell damage in vivo. In addition, SOD is an important antioxidant enzyme in vivo. Numerous studies demonstrated that SOD is a natural OFR-clearing factor, thereby playing an important role as a modulator of the oxidative and antioxidant balance in the body. In the present study, we found that different doses of Munziq can significantly increase SOD and decrease MDA levels in a myocardial I/R rat model, indicating that Munziq has a significant protective effect against MIRI in rats.

In the present study, we also found that the IL-6 and IL-8 levels were significantly decreased after pretreatment with Munziq. IL-6 and IL-8 are produced mainly in monocytes/ macrophages and endothelial cells, inducing T-cell activation, proliferation, and differentiation. Together, IL-6 and IL-8 can induce neutrophils to produce damage to the myocardium.

Our results suggested that the process of $\mathrm{I} / \mathrm{R}$ results in the production of large amounts of IL-6 and IL-8, whereas pretreatment with Munziq results in significant reduction of the levels of these 2 inflammatory factors. Similarly, we found significant decreases in the IL- 6 and IL-8 levels in the Atorvastatin intervention group. These results indicated that Munziq has an obvious anti-inflammatory effect and can inhibit the release of the inflammatory mediators IL- 6 and IL-8, thereby inhibiting neutrophil infiltration and achieving myocardial protection. In addition, moderate or large doses of Munziq had stronger anti-inflammatory effects than Atorvastatin, suggesting that the anti-inflammatory effects of Munziq are superior to those of Atorvastatin.

In conclusion, this study demonstrated a protective effect of different doses of savda Munziq on I/R damage. Further, we verified that Munziq can effectively enhance expression of the protective protein HSP70 in myocardial tissue and decrease the levels of inflammatory factors in serum, which provides evidence for the protective mechanism of Munziq on the myocardium.

\section{ACKNOWLEDGMENTS}

Research supported by the National Natural Science Foundation of China (\#81460755).

\section{REFERENCES}

Abdiryim Y, Halmurat U, Anwar U, Benedicte B, et al. (2011). Abnormal Savda Munziq, an herbal preparation of traditional Uighur Medicine, may prevent1, 2-dimethylhydrazine-induced rat colon carcinogenesis. Evid. Based Complement. Alternat. Med. 2011: 152015.

Abdiryim Y, Halmurat U, Anwar U, Benedicte B, et al. (2012). Ethanol extract of abnormal savda Munziq, a herbal preparation of traditional Uighur Medicine, inhibits Caco-2 cells proliferation via cell cycle arrest and apoptosis. Evid. Based Complement. Alternat. Med. 2012: 926329.

Hamulati U (2008). New theory of Abnormal savda syndrome. Xinjiang Sci. Technol. Press. 54.

Hamulati U, Aidanuer M, Nurmamat A, Abdiryim Y, et al. (2006). Abnormal savda syndrome animal model of crossexamination and rebuttal natural restoration. J. Xinjiang Med. Univ. 29: 910-914.

Hoffman JW Jr, Gilbert TB, Poston RS and Silldorff EP (2007). Myocardial reperfusion injury: etiology, mechanisms, and therapies. J. Extra Corpora Technol. 36: 391-411.

Huang MH, Nguyen V, Wu Y, Rastogi S, et al. (2009). Reducing ischaemia/reperfusion injury through delta-opioidregulated intrinsic cardiac adrenergic cells: adrenopeptidergic co-signalling. Cardiovasc. Res. 84: 452-460.

Krenz M and Robbins J (2004). Impact of beta-myosin heavy chain expression on cardiac function during stress. $J$. Am Coll. Cardiol. 44: 2390-2397.

Li Y and Lv ZG. (2011). Norepinephrine on intracellular expression of CGRP. Zhong Xi Yi Jie He Xin Nao Xue Guan Bing Za Zhi 06. 
Lum H and Roebuck KA (2005). Oxidant stress and endothelial cell dysfunction. Am J. Physiol. Cell Physiol. 280: C719-C741.

Ruiz-Gines JA, Lopez-Ongil S, Gonzalez-Rubio M, González-Santiago L et al. (2006). Reactive oxygen species induce proliferation of bovine aortic endothelial cells. J. Cardiovasc. Pharmacol. 35: 109-113.

Schulze CJ, Wang W, Suarez-Pinzon WL, Sawicka J et al. (2007) Imbalance between tissue inhibitor of metalloproeteinase-4 and matrix metalloproteinase during acute myocardial [correction of myocardial] ischemia-reperfusion injury. Circulation 107: 2487-2492.

Sun ZD, Gao SZ, Mao ZF, Wu Y, et al. (2005). Heat-shock protein expression on immature myocardial protection in rats. $J$. Thoracic Cardiovasc. Surgery 12.21.6.

Yusup A, Upur H, Umar A and Moore N (2004). Protective effects of Munziq and Mushil of abnormal Savda to mitochondrial oxidative damage. Fundam Clin. P. 18: 471-476 